\title{
Research on the Prediction of Second Synchronization in Timing System using Cubic Exponential Smoothing
}

\author{
Haidong Zou \\ China Satellite Maritime Tracking and Control \\ Department \\ Jiangyin, Jiangsu, China \\ E-mail: cnflyfish@foxmail.com \\ Yewei Yang \\ China Satellite Maritime Tracking and Control \\ Department \\ Jiangyin, Jiangsu, China \\ E-mail: yeweiyang@263.com
}

\author{
Zeshui Liu \\ China Satellite Maritime Tracking and Control \\ Department \\ Jjiangyin, Jiangsu, China \\ E-mail: liuzeshui@sohu.com \\ Jingbo Zhang \\ China Satellite Maritime Tracking and Control \\ Department \\ Jiangyin, Jiangsu, China \\ E-mail: zhangjb@tom.com
}

\begin{abstract}
The value of time synchronization between Rubidium clock and GPS clock, which changing with time, was studied by using the time series prediction method. Some issues such as the selection of the smoothing coefficient $\alpha$ and the prediction accuracy problem of linear exponential smoothing, secondary exponential smoothing and cubic exponential smoothing were discussed in this paper. The results showed that the mean absolute error of prediction was minimum if smoothing coefficient $\alpha$ equaled to 0.5 , and the result of cubic exponential smoothing prediction coincided preferably to the measure values.
\end{abstract}

Keywords- Rubidium Clock; Global Position System; Frequency Drift; Time Series Prediction; Smoothing Coefficient

\section{INTRODUCTION}

With the development of the space measurement and control system, the demand for accuracy of the test system is higher. Generally, the accuracy of the timing system determines the accuracy of the whole test system. But for various reasons, all the stations usually use rubidium clock as a frequency standard of punctuality. Through comparis on with the GPS second pulse, the timing system controls the time synchronization accuracy beyond a limit value. If the value exceeds the threshold, then starts artificial intervention with manual second synchronization, to reduce or even eliminate the errors. With heavy of tasks, artificial duty obviously does not meet the demand of tasks. There is an urgent need for using new technology and method to update the existing time synchronization monitoring method, so to improve the efficiency of system operation and maintenance.

The main parameters of the atomic frequency characteristics are frequency stability, frequency accuracy, frequency deviation and frequency drift. It is generally believed that the frequency accuracy is to tell the actual output frequency relative to its nominal frequency deviation of the atomic clock, which does much impact to the punctuality[1]. In the practical applications, the frequency deviation is more important. According to the needs of the communication network construction and development, it should not only know the punctual synchronization accuracy of rubidium clock, also need to predict fluctuations of which for a period of time. Due to influence of the diversity of rubidiu m clock time accuracy, it's more difficult to accurately predict the synchronization precision. In order to avoid the above all sorts of analys is and reduce the difficulty of the research questions, in this paper, the time series prediction method, by using exponential smoothing method for measurement and control station is discussed. The synchronization accuracy of rubidium clock with respect to the GPS second is analyzed in a scientific way.

\section{MODEL OF TIME LAG FOR RUBIDIUM CLOCK}

It is generally considered that the time difference data [2] (usually, the phase data is converted to the time difference data) can be express ed as a formula:

$$
x(t)=x_{0}+y_{0} t+\frac{1}{2} D^{2}+\varepsilon_{x}(t)
$$

Here, $\mathrm{x} 0$ is the in itial time (phase) deviation. And $\mathrm{y} 0$ is the initial frequency deviation. D is to stand for the rate of frequency drift. $\varepsilon x(\mathrm{t})$ is the random variation component of atomic frequency deviation. The frequency drift rate is the intrinsic characteristic of atomic frequency, so it can't be changed. With the formula (1), if the drift of frequency is linear, the influence of which is proportional to the time. To accurately prediction of rubidium clock time $\mathrm{x}(\mathrm{t})$ for a long time, it needs to consider the nonlinear characteristics of the frequency. But in the practical use, there are a lot of factors to be considered with the difference value of time. But with not long time using the timing system, the 
influence of frequency drift about punctuality is relatively small. Then, it can be neglected. At this time, it is the main consideration for the effects of frequency accuracy and external characteristics of timekeeping error. A ground station is using the rubidium clock with GPS cesium clock for time synchronization. Considering the above factors, it can be regarded that the synchronization accuracy varies with time in a linear way. And it can be predicted and analyzed with linear time series prediction method.

\section{METHOD OF TIME SERIES}

The method of time series prediction uses the historical data of target, which are arranged in a sequence with time elapse, to analyze the changing trend over time. Meanwhile, corresponding mathematical model for extrapolation of a quantitative prediction methods are established [3]. It's much convenient in the prediction of objects with much impacts and complex relationships [4]. As to the prediction for timing synchronization precision of rubidiu m clock, it can be used to determine the variation and change trend with time series prediction method.

The exponential smoothing method is one of the methods in time series prediction. It is evolved from the moving average method. By introducing a weighting factor, that is the smoothing coefficient $\alpha$ (in the range $[0,1]$ ), with a certain amount of time series prediction model, it can achieve forecasting future values of time sequence. It's often used in time series forecasting of short cycle trend forecasting [5]. And $\mathrm{x} 0, \mathrm{x} 1, \ldots, \mathrm{xn}$ is the real value of a time series, $\mathrm{S} 1(1), \mathrm{S} 2(1), \ldots, \mathrm{Sn}(1)$ is the forecasted value in the time. The linear exponential smoothing model is:

$$
S_{t}^{(1)}=\alpha x_{t}+(1-\alpha) S_{t-1}^{(1)}
$$

Among them, $\alpha$ is the smooth coefficient. By the formula (2), the forecast value[6] of the next moment is equals to the forecast value of the moment with an error correction value in addition. In general, the forecast accuracy of the linear exponential smoothing method is affected by the smooth coefficient $\alpha$. So it applies to the time series prediction with horizontal trend. Especially, there is a certain trend in the time series, the prediction results tend to lag obviously, and then cause errors. Therefore, a higher order exponential smoothing method is needed.

Look all linear exponential smoothing value $\operatorname{St}(1)(\mathrm{t}=1$, $2, \ldots \mathrm{n})$ as a new time series, and the exponential smoothing method is used once again, secondary exponential smoothing is get with the value of the original time series [7]. It can be used to a linear trend prediction. The model can be expressed as:

$$
S_{t}^{(2)}=\alpha S_{t}^{(1)}+(1-\alpha) S_{t-1}^{(2)}
$$

Similarly, if the $\operatorname{St}(2),(t=1,2, \ldots n)$ is used as a new time series, and the values of cubic exponential smoothing can be get. The model can be expressed as:

$$
S_{t}^{(a)}=\alpha S_{t}^{(2)}+(1-\alpha) S_{t-1}^{(a) j}
$$

The (4) is a second parabolic form. Due to the approximate linear function relationship of rubidium clock synchronization [8], the application of the cubic exponential smoothing method can predict the change trends overtime of time synchronization.

\section{SELECTION OF SMOOTHING COEFFICIENT}

Time synchronization between rubidium clock and GPS second pulse in real-time comparis on is the duty of a ground unit. The values of time synchronization in a certain period of time is sorted out and are specified in

\begin{tabular}{|c|c|}
\hline Time series & Value \\
\hline 1 & -0.12 \\
\hline 2 & 3.76 \\
\hline 3 & 7.74 \\
\hline 4 & 11.56 \\
\hline 5 & 15.82 \\
\hline 6 & 19.75 \\
\hline 7 & 23.68 \\
\hline 8 & 27.94 \\
\hline 9 & 31.58 \\
\hline 10 & 35.60 \\
\hline 11 & 39.75 \\
\hline 12 & 43.48 \\
\hline 13 & 47.44 \\
\hline 14 & 51.68 \\
\hline 15 & 55.80 \\
\hline 16 & 59.77 \\
\hline 17 & 63.62 \\
\hline 18 & 67.52 \\
\hline 19 & 71.84 \\
\hline 20 & 75.60 \\
\hline
\end{tabular}
Table 1.

TABLE I. TIME SYNCHRONIZATION PRECISION (US)

Note: At the moment of time series 1, manual second synchronization with GPS is executed. And the actual time is 09:00am, January 12, 2015. Then the values are recorded for every 1 hour as follows. The value is positive when the local clock is ahead of the GPS clock, and vice versa.

In Table 1, the first 15 values of synchronous precision data are used to predict the 5 values remained. Then the predicted values and the actual values are compared with the mean absolute error, to analyze the accuracy of the prediction results. The smaller the mean absolute error is, the higher the prediction accuracy is .

Matlab is used to calculate the accuracy prediction of the second synchronization. By setting the initial smoothing value $\mathrm{S} O(3)=\mathrm{S} 0(2)=\mathrm{S} 0(1)=(\mathrm{x} 0+\mathrm{x} 1+\mathrm{x} 2) / 3$. Generally, the value remains constant in the whole prediction. Key to the forecast is the value selection of the smoothing coefficient, which affects the accuracy of forecasting. By empirical method[9-10], when the value of time series trend stability, choosing smaller $\alpha(0.05 \sim 0.20)$; slight fluctuations, but the long-term trend changes little, choosing lightly larger $\alpha(0.1 \sim 0.4)$; with fluctuations, and the long-term trend of the magnitude changes larger, and with a clear and sharp rise or decline, then select a large 
$\alpha(0.5)$; when the time series data appear sharp rise or a sharp decline in the trend, then $\alpha$ takes a larger value (0.5 1). As to the prediction of second synchronization, the mean absolute error (MAE) is calculated with different values of $\alpha$ as:

$$
\varepsilon=\sqrt{\frac{1}{n} \sum_{t=1}^{n}\left(x_{t}-\hat{x}_{t}\right)^{2}}
$$

is prediction value in $\mathrm{n}$ time series, and $\mathrm{xt}$ is the measured value. The results of the cubic exponential smoothing method for the prediction of different values are shown in Table 2.

TABLE II. MAE OF THE PREDICTION WITH DIFFERENT VALUES OF SMOOTHING COEFFICIENT

\begin{tabular}{|c|c|}
\hline$\alpha$ & $\varepsilon$ \\
\hline 0.1 & 2.54 \\
\hline 0.2 & 1.98 \\
\hline 0.3 & 0.78 \\
\hline 0.4 & 0.32 \\
\hline 0.5 & 0.29 \\
\hline 0.6 & 0.32 \\
\hline 0.7 & 0.36 \\
\hline 0.8 & 0.40 \\
\hline 0.9 & 0.44 \\
\hline
\end{tabular}

Table 2 shows that when $\alpha$ is close to zero, the weighting of past time series decreasing slower, namely the past data can affect the prediction. And if $\alpha$ is close to 1 , the weight of time series decreasing faster, namely the past data affect the prediction a little. In addition, when $\alpha=0.5$, the mean absolute error of the prediction is minimum. Therefore, in the prediction of rubidiu m clock, $\alpha=0.5$ is used in the cubic exponential smoothing method.

\section{PREDICTION RESULT ANALYSIS}

For linear exponential smoothing, secondary exponential smoothing and cubic exponential smoothing methods, the results of the prediction are shown in Figure 1.

From Fig. 1, the prediction of the linear exponential smoothing method has obvious hysteresis, and the error of the value to the true value is relatively large. The secondary exponential s moothing method cannot simulate the actual trend with time because of its predictive model for linear equation. The cubic exponential smoothing method can simulate the real time variation accurately. But for the limitation of the time series method, which is based on changes with time, there are still some errors between the forecast value and the true value. And the error changes with time basically unchanged.

Setting $\alpha=0.5$, and using the measured data of the time series in table 1 , to predict the second synchronization data in the next 5 time series, as shown in Fig. 2. Using high precision of cubic exponential s moothing method to predict, which can effectively eliminate the influence of random fluctuations in historical data, better simulate the difference with the time variation of the actual trend.

The Output of Four Methods

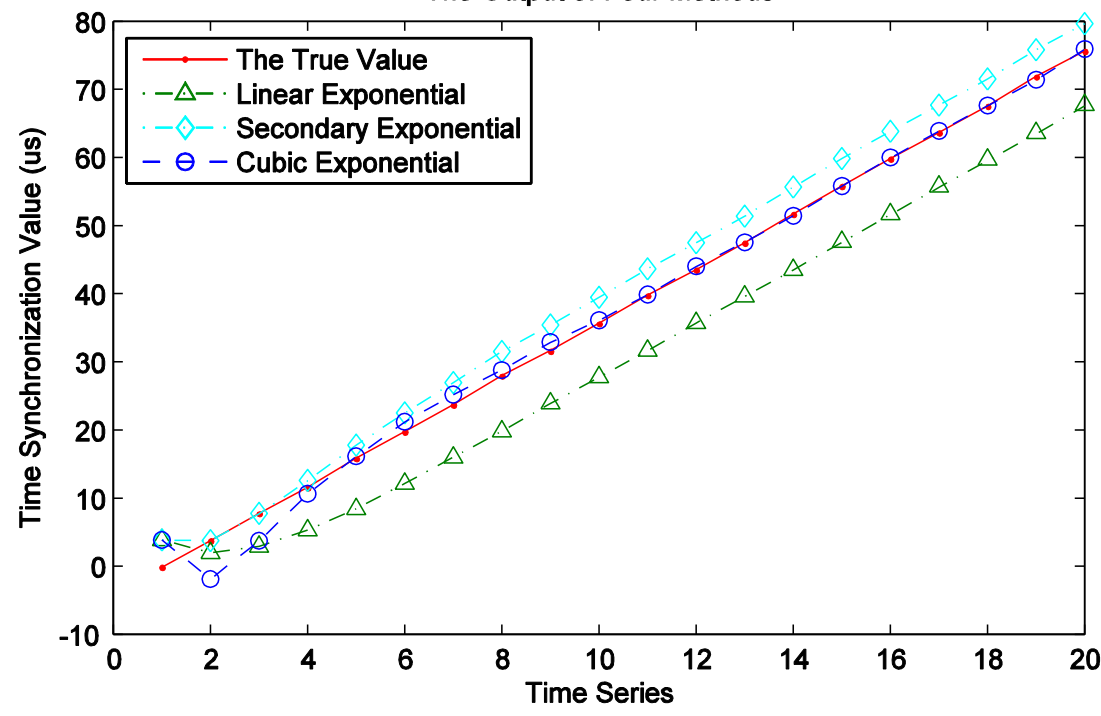

Figure 1. Comparison curves of 3 smoothing methods and the true observation values. 


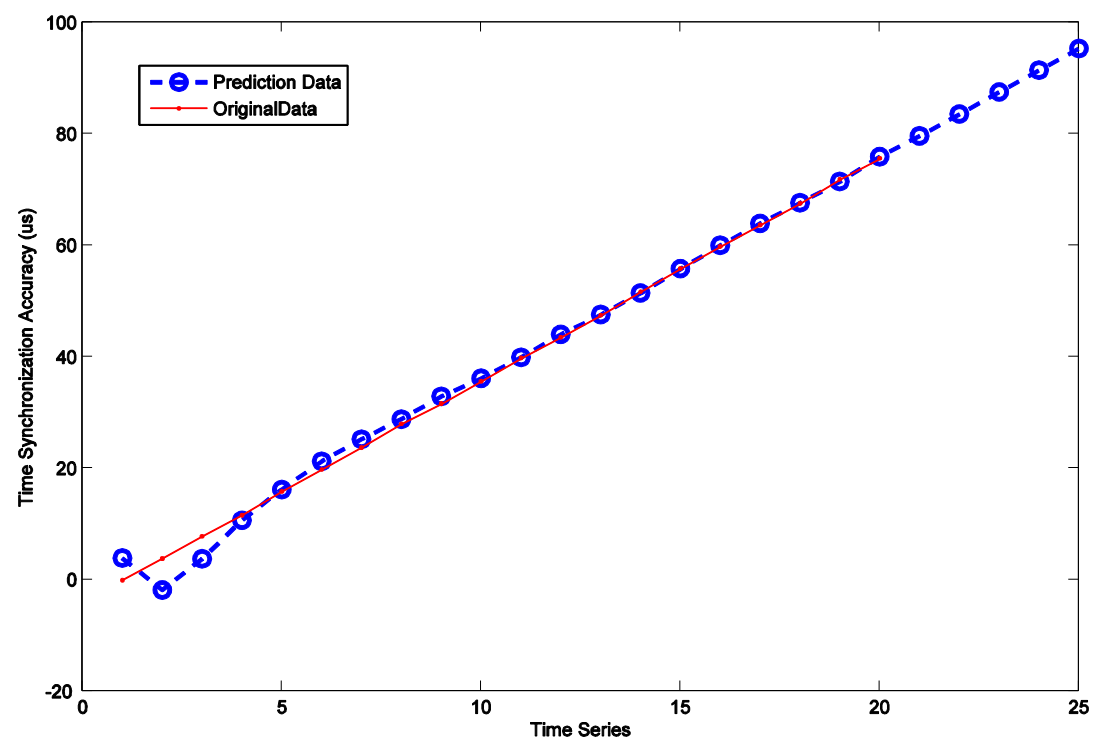

Figure 2. Five results of prediction with cubic exponential smoothing method.

\section{CONCLUSIONS}

The cubic exponential smoothing method is discussed to predict the accuracy of synchronization for a rubidium clock in this paper. By selecting different smoothing coefficient, the values of both predicted and measured are compared. Thus the best smoothing coefficient is got in order to improve the accuracy of prediction. However, for the limitation of the time sequence method, a higher precision of prediction can be got only in the gentlest on time series variation. Therefore, the exponential smoothing method is used to forecast the short-term second synchronization accuracy, and the MAE is small. In order to further predict the long-term variation trend of the accuracy of the second synchronization, it is necessary to adopt a more intelligent forecasting method.

\section{REFERENCES}

[1] Guo Hairong, "Analysis of the frequency drift of navigation satellite with atomic clocks," Global positioning system, 2007.06.

[2] Tong Baorun. "Timing System," National Defence Industry Press, 2004.
[3] Wang Liming, Wang Lian, "Application of time series analysis," Fudan University press, 2009.

[4] Lu Jiqiang, Xu Feng, "Research of landslide forecast model based on the exponential smoothing and regression analysis[J]. Journal of Wuhan University of Technology, 2011.10.

[5] Bonsdorff.H, "A comparison of the ordinary and a varying parameter exponential smoothing," J Appl Prob,1989.

[6] Song Fang, "Studies on futures forecasting system based on genetic simulated annealing and cubic exponential smoothing," Tianjin University, 2012.

[7] Hyndman, Rob J., "Forecast ing with exponential smoothing : the state space approach," Springer , 26.1, 2008, pp.204-205.

[8] Hyndman, Rob J., M. Akram, and B. C. Archibald, "The admissible parameter space for exponential smoothing models," Annals of the Institute of Statistical Mathematics 60.2, 2008, pp.407-426.

[9] Zhang, Qiong, "Building Material Price Forecast Based on the Three Exponential Smoothing Method," Railway Engineering Cost Management, 2013.

[10] F., Pozzi, M. T., and A. T, "Exponential smoothing weighted correlations," The European Physical Journal B - Condensed Matter and Complex Systems, 85.6, 2012,pp.1-21. 